\title{
BMJ Open Patient navigators facilitating access to primary care: a scoping review
}

\author{
Annette Peart, ${ }^{1}$ Virginia Lewis, ${ }^{2}$ Ted Brown, ${ }^{3}$ Grant Russell ${ }^{1}$
}

To cite: Peart A, Lewis V, Brown T, et al. Patient navigators facilitating access to primary care: a scoping review. BMJ Open 2018;8:e019252. doi:10.1136/ bmjopen-2017-019252

- Prepublication history and additional material for this paper are available online. To view these files, please visit the journal online (http://dx.doi. org/10.1136/bmjopen-2017019252).

Received 21 August 2017 Revised 6 February 2018 Accepted 12 February 2018

Check for updates

${ }^{1}$ Southern Academic Primary Care Research Unit, Department of General Practice, School of Primary and Allied Health Care, Monash University, Notting Hill, Australia

${ }^{2}$ Australian Institute for Primary Care and Ageing, La Trobe University, Melbourne, Australia ${ }^{3}$ Department of Occupational Therapy, Monash University, Frankston, Australia

Correspondence to

Annette Peart;

annette.peart@monash.edu

\begin{abstract}
Objective Patient navigators are a promising mechanism to link patients with primary care. While navigators have been used in population health promotion and prevention programmes, their impact on access to primary care is not clear. The aim of this scoping review was to examine the use of patient navigators to facilitate access to primary care and how they were defined and described, their components and the extent to which they were patient centred.

Setting and participants We used the Arksey and 0'Malley scoping review method. Searches were conducted in MEDLINE, Embase, ProQuest Medical, other key databases and grey literature for studies reported in English from January 2000 to April 2016. We defined a patient navigator as a person or process creating a connection or link between a person needing primary care and a primary care provider. Our target population was people without a regular source of, affiliation or connection with primary care. Studies were included if they reported on participants who were connected to primary care by patient navigation and attended or made an appointment with a primary care provider. Data analysis involved descriptive numerical summaries and content analysis. Results Twenty studies were included in the final scoping review. Most studies referred to 'patient navigator' or 'navigation' as the mechanism of connection to primary care. As such, we grouped the components according to Freeman's nine-principle framework of patient navigation. Seventeen studies included elements of patient-centred care: informed and involved patient, receptive and responsive health professionals and a coordinated, supportive healthcare environment.

Conclusions Patient navigators may assist to connect people requiring primary care to appropriate providers and extend the concept of patient-centred care across different healthcare settings. Navigation requires further study to determine impact and cost-effectiveness and explore the experience of patients and their families.
\end{abstract}

\section{INTRODUCTION}

Primary care is the first level of access to healthcare, delivered in the community most often by family physicians or general medical practitioners. However, not all people access primary care that best meets their healthcare needs, where and when they need it. Some people, such as those living in poverty, with a long-term disability, from a culturally and linguistically diverse background
Strengths and limitations of this study

- This is the first scoping review to explore how patient navigators are defined, described and used to facilitate access to primary care for people without an affiliation to a primary care provider.

- It is a comprehensive overview of sources covering peer-reviewed and grey literature.

- Sources were included only if the outcome of the navigation was reported; sources describing patient navigation without reporting of outcomes were excluded.

- The inclusion of a description of the patient centredness of the sources is a unique addition to this review of patient navigators.

or located in rural and remote areas, have difficulty accessing primary care services and resources. $^{1-4}$

Access to healthcare is the opportunity to reach and obtain appropriate healthcare in situations of perceived need. ${ }^{5}$ Access to primary care is important to reduce healthcare disparities, mortality, morbidity, hospitalisation rates and healthcare costs. ${ }^{6-9}$ Recent reforms to primary care have focused on trialling new processes and models of care to improve access. ${ }^{10}$ These include integrated care models, after-hours telephone consultations, walk-in centres and nurse-led initiatives. However, disparities in care remain for many, such as people having low literacy and numeracy, cognitive deficits, being a member of a marginalised group or not understanding the need for primary care. ${ }^{11}$

A new approach to improve access to primary care is patient navigation, a process where a person (navigator) engages with a patient to determine barriers to care and provides information to improve access to components of the health system, not just primary care. ${ }^{12}$ A patient navigator has been described as a type of 'broker' who uses a biopsychosocial approach to provide a range of instrumental and relational functions and processes $^{1314}$ to support patients to access primary care and directly identify providers willing to treat vulnerable people requiring 
care. ${ }^{15}$ Patient navigator tasks can include educating patients about early symptoms of cancer (in preventive care) or facilitating and coordinating appointments with providers to improve access to a regular primary care provider. Originating in the 1990s, Freeman developed patient navigation as a strategy to reduce barriers to breast cancer care in Harlem, New York. ${ }^{16}$ Since then, patient navigators have been used for the screening of various cancers and through the cancer care continuum, with mixed success. ${ }^{17-27}$ In primary care, navigators may have a role in improving access and coordination of care, especially for vulnerable populations whose access to care may be compromised by a range of geographic, demographic, socioeconomic or cultural characteristics. ${ }^{28}$

Patient-centred care is a core element of high-quality primary care, facilitates access to appropriate care $^{11}$ and has been identified as one of six areas of focus for improving healthcare systems. ${ }^{29}$ In primary care, patient-centred care consists of interactions and relationships between providers and patients to share information, explore values and preferences, facilitate access to appropriate care, and address healthcare disparities. ${ }^{30} 31$ While numerous frameworks of patient-centred care have been described, ${ }^{32}$ Epstein's ${ }^{11}$ succinct model of patient-centred care comprising an informed and involved patient, receptive and responsive health professionals and a coordinated, supportive healthcare environment, sits well within the context of patient navigation and its extension beyond the patient-clinician relationship to the setting in which care is delivered.

While navigators have been used in population health promotion and prevention programs, ${ }^{33} 34$ there has been recent interest in their use in facilitating access to primary care for vulnerable people without a regular primary care provider. $^{28}$ Understanding the components of these programmes can assist those interested in designing or implementing similar programmes. Therefore, we performed a scoping review of the use of patient navigation to facilitate access to primary care. Given its importance and relevance to navigation, we included an additional focus on the extent to which identified patient navigation interventions were patient centred.

\section{METHODS}

We chose the scoping review method to map the extent, range and nature of published research on the use of patient navigation to further understand how it links people to primary care. ${ }^{35}$ When compared with systematic reviews, scoping reviews address broader topics and are less reliant on detailed research questions or quality assessments. ${ }^{35}$ The work was structured around the five stages of the Arksey and O'Malley framework: (1) identify the research question, (2) identify relevant studies, (3) study selection, (4) chart the data and (5) collate, summarise and report the results. The review was also informed by Levac et al $\mathrm{s}^{36}$ refinements to Arksey and O’Malley's framework.
Stage 1: identify the research question

Patient navigation has been defined as a "process, by which an individual, a patient navigator, guides patients in overcoming barriers to healthcare services access to facilitate timely access to care' ${ }^{37}$ We expanded this definition to include a patient navigator as a person or process creating a connection or link between a person needing primary care and a primary care provider.

Our target population was people without a regular source of or affiliation or connection with primary care. The outcome of interest was the person needing care attended an appointment or made contact with the referred primary care provider. These definitions helped us to clarify the focus of the review, confirm the inclusion criteria adopted and establish parameters for the search strategy. ${ }^{36}$ This review did not focus on the impact or effectiveness of patient navigation programmes in this context. We asked three questions to guide the scoping review:

1. How have patient navigators been defined and described in connecting people who are unattached to primary care to a primary care provider for regular care?

2. What are the components of these patient navigation programmes?

3. To what extent has patient centredness been incorporated into the design, implementation and analysis of patient navigation programmes?

\section{Stage 2: identify relevant studies}

We identified relevant studies through a search of electronic databases, grey literature and reference lists of key articles sourced (online supplementary file 1).

A three-step search strategy was used. First, we undertook an initial limited search of MEDLINE, Embase and Cumulative Index to Nursing and Allied Health Literature (CINAHL) using terms and variants of 'navigator', 'broker', 'link worker' and 'community health worker'. We analysed the text in the titles and abstracts of retrieved studies and index terms used to refine key terms. The terms most common were related to navigation, linkage and access to care. We completed a second search of the same databases and extended the search to include related medical and social science databases and grey literature using the key terms and variants (table 1) identified by the initial search strategy (online supplementary file 2).

Finally, we checked the reference lists of all identified studies (and their citations) for additional studies.

\section{Stage 3: study selection}

Inclusion criteria were applied as a basis for which studies were considered relevant to the review questions. Studies were included if they:

- were published in English from January 2000 to May 2016. The start date of 2000 reflects the increasing interest in patient-centred care in the last two decades. Reforms of primary care commenced around this 


\begin{tabular}{|c|c|}
\hline $\begin{array}{l}\text { Concept, programme } \\
\text { or intervention }\end{array}$ & Setting \\
\hline $\begin{array}{l}\text { Navigator/navigation } \\
\text { Patient navigator/navigation } \\
\text { Peer navigator/navigation } \\
\text { Broker } \\
\text { Health broker } \\
\text { Health services broker } \\
\text { Community health worker } \\
\text { Community navigator/ } \\
\text { navigation } \\
\text { Lay health worker } \\
\text { Linkage to care }\end{array}$ & $\begin{array}{l}\text { Community health } \\
\text { Family practice/practitioner } \\
\text { General practice/practitioner } \\
\text { Primary care } \\
\text { Primary healthcare }\end{array}$ \\
\hline
\end{tabular}

time $^{29}$ along with the emergence of navigator-type approaches $^{38}$;

- reported on patients who did not have a regular source of primary care (provider or practice);

- connected patients to primary care by a process (eg, navigation) or a person (eg, navigator);

- reported an outcome of patients attending or making at least one appointment with primary care providers.

We excluded studies if they originated in countries who were not members of the Organisation for Economic Cooperation and Development (OECD), as their primary care systems differ significantly from those of OECD countries. Other exclusion criteria were applied to studies where:

- patients lived in residential care, or incarcerated with no imminent release date, as their primary care needs were assumed to be met by institutional providers;

- a navigator was attached to a primary care provider or practice as this indicated the patient was already connected to primary care;

- a navigator referred patients to health screening or assessment services only and not to a primary care provider.

AP reviewed titles and abstracts of studies, and GR independently reviewed abstracts where there was uncertainty for inclusion.

\section{Stage 4: chart the data}

Data extracted were entered into a template developed in Microsoft Excel specifically for this review. Information on authors, year of publication, study location and context, aims or purpose of the research, study type or design, population and sample size, methodology, conceptual model, intervention type and duration, measures used and key findings were recorded on this form. We also extracted data relevant to the research questions: definitions and descriptions of navigators, components of navigator programmes and elements of patient-centred care. Charting the data was an iterative process ${ }^{36}$ that we updated as studies revealed useful data categories. Studies were reviewed a number of times to ensure all relevant data was captured.

\section{Stage 5: collate, summarise and report the results}

We collated the data using a Microsoft Excel spreadsheet. Excerpts of text were coded deductively by AP to identify concepts and themes related to the research questions. GR checked the coding scheme and the themes raised.

\section{RESULTS}

Our initial search terms generated 6355 records from electronic databases and grey literature (figure 1). We removed 664 duplicates, leaving 5691 records to be screened. Of these, 5613 records were excluded based on the title and/or abstract review, as they were not relevant to the question, did not meet inclusion criteria or originated in non-OECD countries. Of the remaining 78 records, full-text review excluded 44 where participants were not linked to primary care and 16 where participants already had a primary care provider or did not indicate a need for primary care. We searched references and citations of the remaining 18 records, adding two additional studies. This resulted in 20 selected for inclusion in the scoping review. The selection process is shown in the flow chart (figure 1).

Of the 20 included studies, three reported on the same randomised controlled trial at different phases. ${ }^{39-41}$ These three studies were counted as unique studies as each reported on different elements of the same trial: preliminary findings, qualitative analysis of interviews and longitudinal findings.

Eleven studies were descriptions or evaluations of programmes, eight were intervention studies and one was a retrospective study. Thirteen were programmes based in emergency departments, six were community-based programmes and one was delivered in an inpatient setting. All studies were conducted in the USA. Table 2 outlines characteristics of the included studies.

\section{Patient navigators: definition and descriptions}

One study defined patient navigation as a "process, by which an individual, a Patient Navigator, guides patients in overcoming barriers to health care services access to facilitate timely access to care'. ${ }^{37}$ The studies provided either a description of a navigator (person) or, for three of the studies, navigation process. ${ }^{42-44}$ Descriptions varied in detail and often consisted of the type of person recruited as a navigator, the tasks they performed and the training provided (table 2 ).

\section{Patient navigation programme components}

All of the studies outlined components of their programmes; four provided detailed descriptions. $^{39-41} 45$ We grouped programme components according to Freeman's consensus-based nine-principle framework of patient navigation, originally developed in response to the expansion of patient navigation as a community-based intervention. ${ }^{16} 4647$ These principles have been widely used in patient navigation programmes. Each of these principles is outlined below with examples 


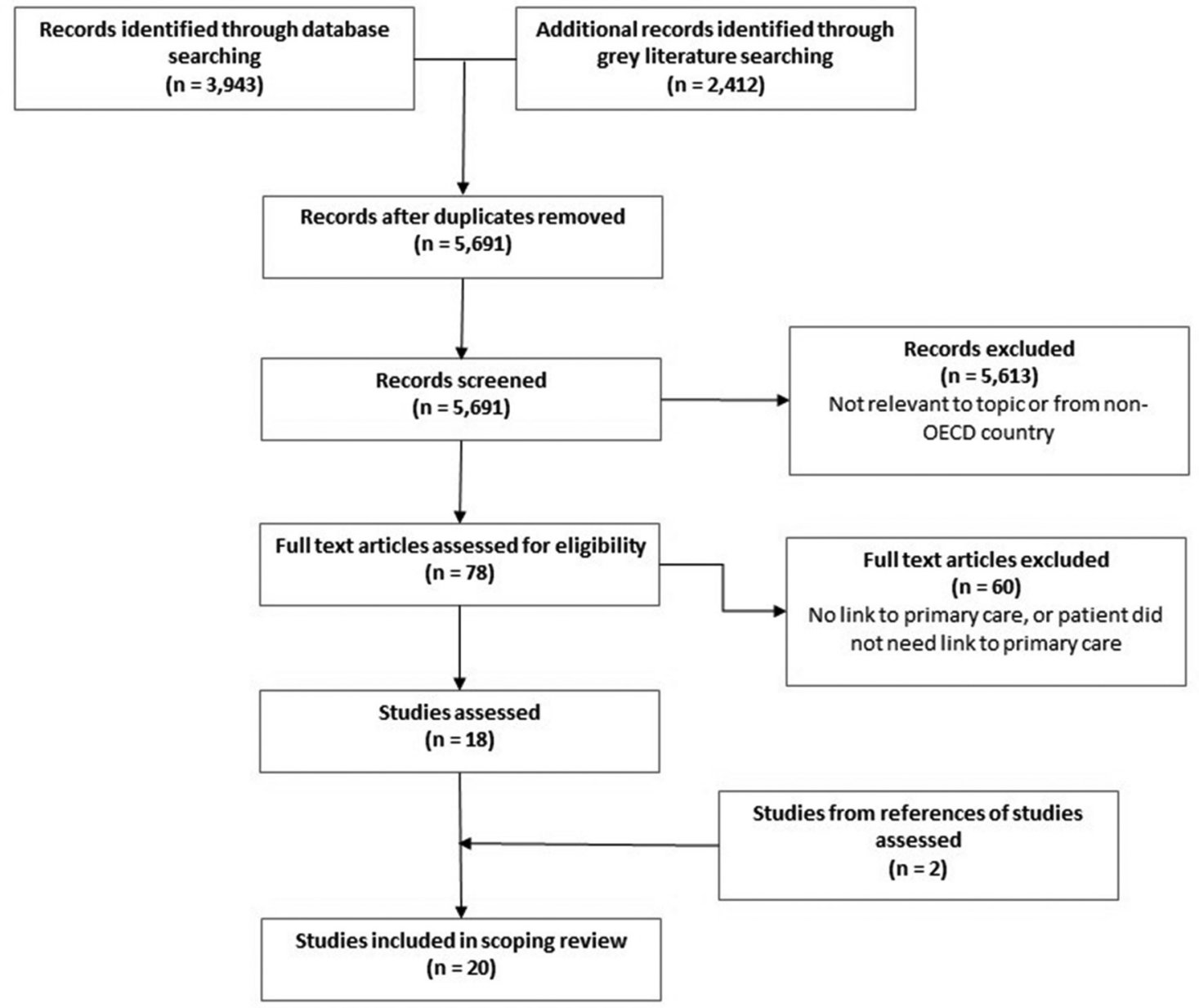

Figure 1 Flow of study selection. OECD, Organisation for Economic Cooperation and Development.

from the studies selected that included sufficient information to inform each principle in the framework.

Principle 1: patient-centred healthcare service delivery model Seventeen of the studies outlined aspects of patient-centred care. This will be discussed further in the section addressing research question three.

Principle 2: integration of a fragmented healthcare system

This principle relates to a patient experiencing a seamless, timely flow through the continuum of care. ${ }^{16}$ We grouped another principle (principle 8: connect disconnected healthcare systems) here with principle 2 , as the two are similar concepts, and this has been done previously. ${ }^{48}$ All studies in our scoping review reported on these principles grouped together. Two examples of integration in our scoping review were assisting patients to understand the entire health system, ${ }^{49}$ and linking the emergency department with a primary care provider, as well as to community dental, mental health, substance abuse and other social services. ${ }^{50}$

\section{Principle 3: elimination of barriers}

This principle is most effectively carried out through relationships with patients. ${ }^{16}$ While removing barriers to accessing primary care appears implicit in a navigator programme, not all studies provided detail of what the barriers were and how they were addressed. One exception of note is the Step on It! intervention at JFK International Airport, which focused on the barriers taxi drivers faced. This intervention went to the airport holding lot, assisted drivers to locate providers with flexible hours, culturally and linguistically appropriate models of care and at low-cost. ${ }^{51}$ Another study described a programme that helped adults with sickle cell disease find primary care. ${ }^{52}$ The barriers addressed included patients not understanding why they needed a primary care provider when they already had a specialist, low literacy, difficulty filling out forms and forgetting appointments. These navigators used motivational interviewing to identify further barriers and help patients set priorities beyond accessing primary care. $^{52}$

\section{Principle 4: clear scope of practice}

Three studies provided detail about the role and responsibilities of the navigator. ${ }^{37452}$ The most detailed of these was a randomised clinical trial by Kangovi et al, ${ }^{45}$ providing a website link (http://chw.upenn.edu) containing protocols for recruitment, training and standardised work practices for navigators, organisational directors and managers. 


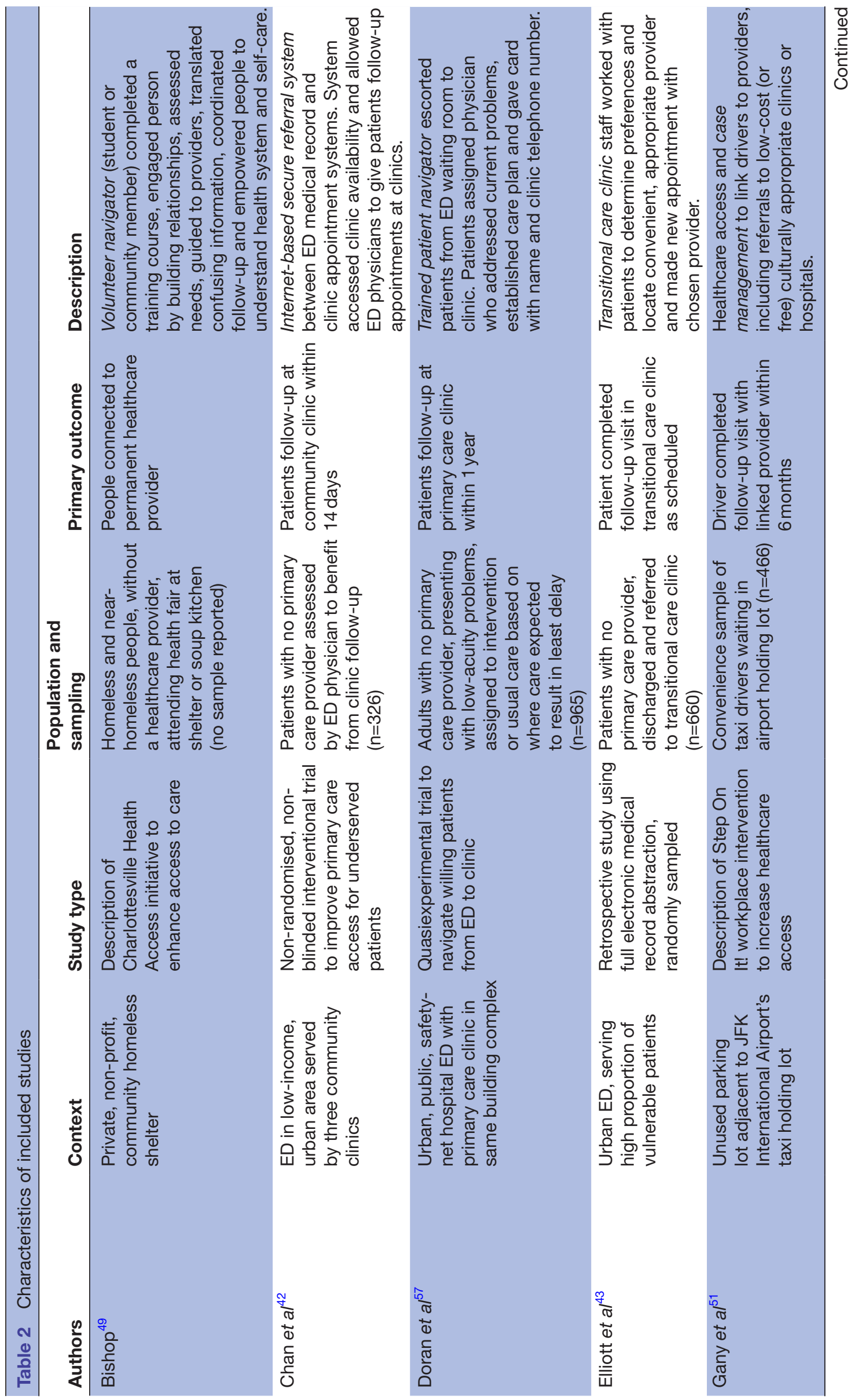




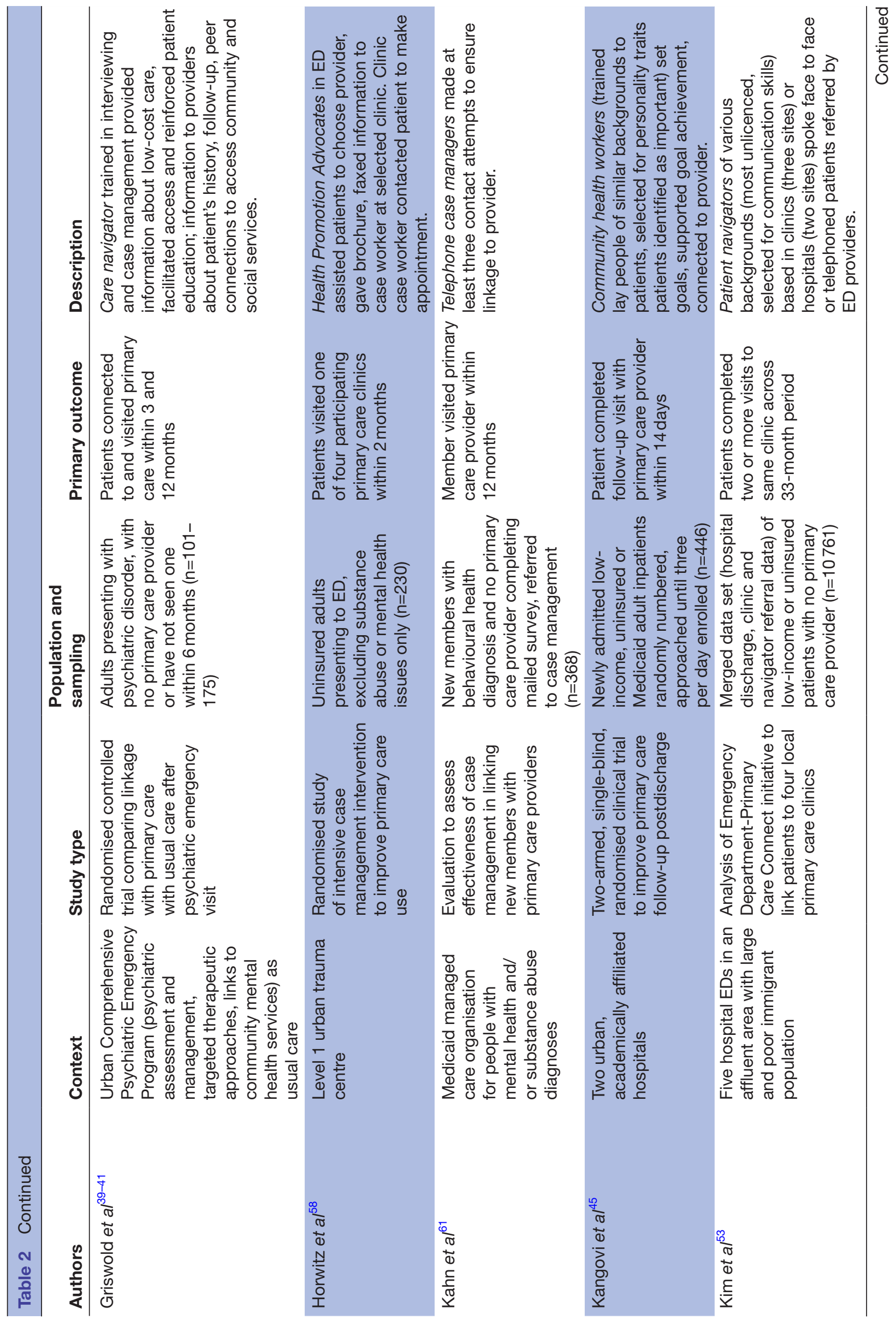




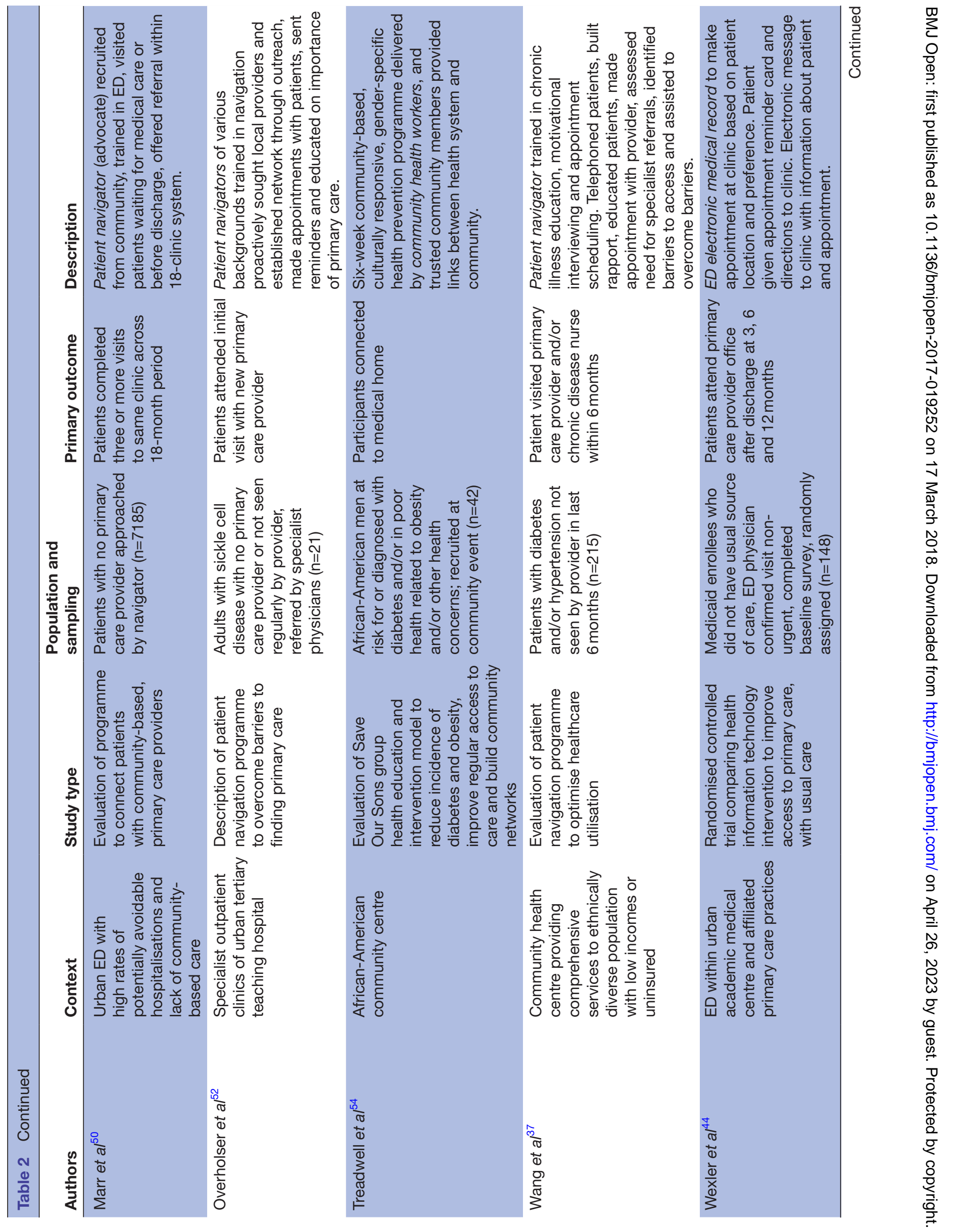




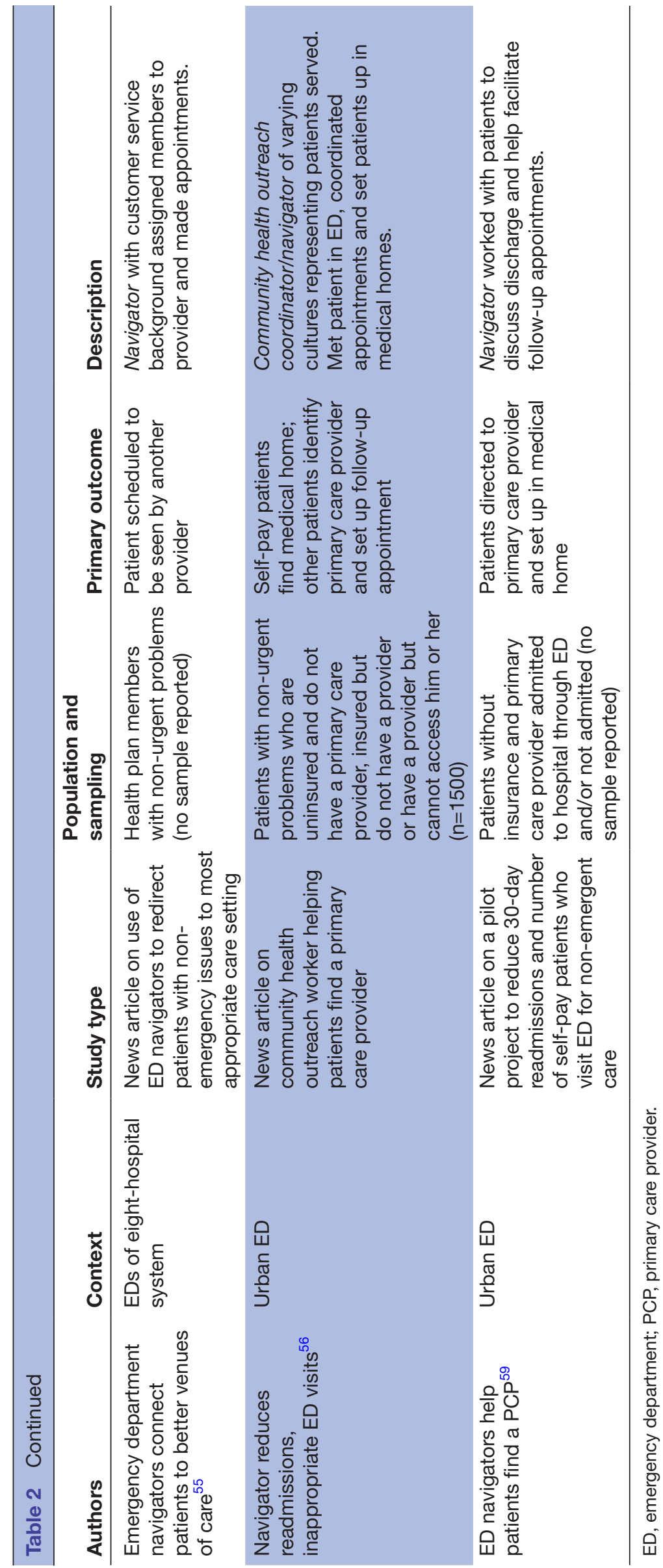


Kangovi et $a t^{45}$ created a community health worker model and tested its effect on posthospital outcomes among general medical inpatients. This was based on qualitative participatory action research and had detailed protocols including standardised work practices in three stages: goal setting, goal support and connection with primary care. A substantial component was to build relationships with patients to help set goals for recovery, develop an individualised action plan and liaise between the patient and inpatient care team. The worker provided tailored support based on the patient goals. Patients were connected to primary care and coached to make and attend appointments independently. Provider resources included a discharge summary and the patient's action plan taken to the appointment.

\section{Principle 5: cost-effective}

None of the studies evaluated the cost-effectiveness of their programme.

\section{Principle 6: defined level of skill}

Nine studies provided information on the skill level required of the navigators. ${ }^{39} 45495052-55$ This ranged from volunteers with inhouse training, staff with customer service backgrounds, to college-accredited navigators. They were trained on topics such as navigation processes, disease-specific content such as diabetes education or motivational interviewing. Similarly, seven studies presented strategies intentionally used to inform the development of resources to support the navigation intervention, including a needs assessment, ${ }^{49}{ }^{56}$ software development, ${ }^{42}$ community-based participatory action research ${ }^{45} 5154$ and provider collaboration to develop and test navigation mechanisms. ${ }^{50}$

\section{Principle 7: defined beginning and end}

Eleven studies outlined definite points at which navigation began and ended. ${ }^{37} 42-455051$ 56-59 Entry usually involved meeting a patient (eg, in the emergency department or on a hospital ward) to schedule an appointment. End points of the interventions included 'patient has an appointment made' or 'patient sees provider'.

\section{Principle 8: connect disconnected healthcare systems}

This principle was combined with a similar principle (principle 2: integration of a fragmented healthcare system) for the purposes of this review.

\section{Principle 9: coordinated system}

This principle relates to having an assigned coordinator to oversee all aspects of the intervention. ${ }^{16}$ This was evident in two studies: where navigators served as executive officers on a governing board ${ }^{49}$ and were supervised by a social worker as well as having weekly team meetings. ${ }^{45}$

\section{Patient navigation: patient centredness}

Our third question for this review was, 'To what extent has patient-centredness been incorporated into the design, implementation and analysis of patient navigation programs?' We focused on the three factors on which patient-centred care depends: informed and involved patient, receptive and responsive health professionals and a coordinated, supportive healthcare environment. ${ }^{11}$ Seventeen studies included at least one of the three factors. Table 3 indicates the number of studies and some examples of approaches to patient-centred care for each of the three factors. The columns of the table indicate whether patient centredness was included in the design, implementation or analysis phase of patient navigation programmes.

The Kangovi et $a l^{45}$ study had an explicit patient-centred focus. The intervention prioritised relationship building with patients through goal setting and development of action plans, liaising with inpatient staff to ensure the patient's goals were at the forefront and giving the action plan to a provider the patient chose based on needs and preferences.

Similarly, in the three studies reporting the same randomised controlled trial, Griswold et $a l^{89-41}$ used a care navigator to connect patients with a history of psychiatric crisis to primary care. The navigator built relationships by meeting with patients routinely while admitted and also at primary care appointments and maintaining regular contact via phone or in person. The navigator would take the patient to the appointment and reinforce any education provided. Patients were informed of low-cost clinics, and further assistance was provided through coordinating follow-up and connecting patients to peer and social services. Provider resources included information to clinics on discharge diagnosis, medications and mental health treatment site referral.

Other studies included the three factors yet did not explicitly state patient centredness as a driver.

\section{DISCUSSION}

Our scoping review identified 20 studies that used patient navigation to facilitate access, and connect vulnerable patients without regular primary care, to a primary care provider. All except three studies used a person to connect the patient to a provider; the remaining three used a navigation process. Most programmes described components that could be included in a framework of patient navigation, and 17 of the 20 studies included factors inherent to patient-centred care in their design, implementation or analysis.

The level of detail in descriptions of the studies varied; this variation has been reported elsewhere. ${ }^{60}$ In the studies included in this review, different terms were used for the same role: patient or care navigator, advocate, case manager or community health worker, for example. This presents challenges in clearly characterising navigators and understanding what they do. Similarly, while there is no generally accepted definition of patient navigation, there is a call for descriptions of the tasks navigators do and the networks of contacts they use to support their 
Table 3 Examples of patient centredness

\begin{tabular}{|c|c|c|c|c|}
\hline Patient-centred care factor & $\begin{array}{l}\text { Design phase } \\
\text { examples }\end{array}$ & $\begin{array}{l}\text { Implementation phase } \\
\text { examples }\end{array}$ & $\begin{array}{l}\text { Analysis phase } \\
\text { examples }\end{array}$ & $\begin{array}{l}\text { Total } \\
\text { studies* }\end{array}$ \\
\hline $\begin{array}{l}\text { Patients informed and involved } \\
\text { in their care }\end{array}$ & $\begin{array}{l}\text { Two studies: } \\
\text { user-friendly and } \\
\text { culturally sensitive } \\
\text { health materials; } \\
\text { bilingual, bicultural } \\
\text { community members }\end{array}$ & $\begin{array}{l}17 \text { studies: provided information } \\
\text { to patient on difference between } \\
\text { emergency and primary care; } \\
\text { identified barriers to access and } \\
\text { help to overcome barriers }\end{array}$ & No studies & 19 \\
\hline
\end{tabular}

${ }^{*}$ Some studies included more than one instance of the patient-centred factor in more than one phase of the intervention.

actions. ${ }^{60}$ Valaitis $e t a l^{28}$ described the specific activities undertaken by patient navigators: facilitating access to health-related programmes, promoting and facilitating continuity of care, identifying and removing barriers to care and effective and efficient use of the health system. Our findings add to these activities: a key feature of patient navigation to facilitate access to primary care is a relationship-based approach, informing and involving patients in connecting them to care.

The studies in this scoping review included elements that seemed to match the components of Freeman's patient navigation framework. This indicates the framework may be generalisable to the tasks of connecting vulnerable people without a primary care provider to regular care. An evaluation of these principles used in 10 self-identified breast cancer navigation programmes using observation of patient navigator activities found the programmes were consistent with individual-level principles (eg, eliminating barriers, patient-centred care and integration of care); however, programme-level principles (eg, skill level, scope of practice and coordinated system) were not consistent across the programmes. We did not examine this level of detail for our scoping review, however, can see a role for this type of observation-based study to further contribute to this field. ${ }^{48}$ Generally, programmes adhered to published criteria for patient-centred care. ${ }^{11}$ Although not overtly stated as an aim, almost all studies incorporated at least one of the three patient-centred care factors: an informed and involved patient, receptive and responsive health professionals and a coordinated, supportive healthcare environment. We found these mostly in the implementation of the programmes to a lesser degree in the design phase and mentioned in only three studies in the analysis. Our assertion that a navigator working with patients unattached to primary care is patient centred, with a focus on connections and relationships, has some merit.

This scoping review has several limitations. Although a scoping review is iterative and involves revisiting the research question and key terms during searches, our search strategy may have missed studies that reported on interventions not designed to connect people to primary care but where this connection may have been a secondary outcome of the intervention (eg, access to information on cancer screening may have prompted participants to link in with a primary care provider). Additionally, information in the title and abstracts of such studies may not have referred to primary care. This approach, however, allowed us to undertake a more targeted review. Similarly, while our search strategy sought to include all terms we determined could be synonymous with patient navigation, we may have missed studies where different names were used for the same function.

Studies where there was no indication patients attended a primary care appointment were not included in our review. While this strategy contributed to a more focused search, studies that reported the implementation of programmes but not outcomes are missing. All 
of our included studies originated in the USA, which we acknowledge would impact on generalisability. These limitations highlight the need for consistent documentation of processes to improve access to care and the outcomes measured.

We did not look for or report on the effectiveness of the interventions or programmes in our included studies. While we are unable to report on the impact, we consider our approach to looking at descriptions and uses of patient navigation in this specific context of connection to primary care, with a focus on patient-centred care, is consistent with the current focus on patient-reported outcome measures and acknowledging the patient experience of care.

This paper contributes to the discussion of access to primary care by considering patient navigation to connect vulnerable populations to providers in three ways. First, we aligned components of the patient navigation studies reviewed to an existing generic navigation framework. This framework appears to be appropriate for considering navigators facilitating access for people without a primary care provider to regular care. Second, a relational approach acts as the backdrop to connecting vulnerable people to care, based on principles of patient-centred care. Finally, in the absence of a consistent definition of patient navigation in facilitating access to primary care, we have added to an existing description of patient navigation activities, which will assist clinicians and researchers to design and implement similar programmes.

\section{Implications for practice}

The studies included in the review used navigators in a range of settings, from emergency departments, inpatient wards, outpatient services and in the community. Most of these studies demonstrate established principles of patient navigation and use a patient-centred approach, particularly when using a navigator (person) rather than a process, such as an electronic system. For providers and organisations wanting to link vulnerable people to primary care in a patient-centred way, navigators may assist in this process.

\section{Future research}

Analysis of cost effectiveness, while not a focus of this review, was nevertheless absent in the cited studies. As the concept of navigator continues to show promise, further research is required to measure impact and give direction to settings interested in using this intervention. For example, the link between patient navigation principles and outcomes of interest require further exploration.

\section{CONCLUSION}

Patient navigators may be used across healthcare settings to improve access to primary care. Navigators are inherently patient-centred due to their relational approach and ability to connect people to primary care. Interventions to improve access to primary care require further study to determine their impact and cost-effectiveness.

Acknowledgements We wish to acknowledge the peer reviewers of the original version of this paper who provided valued and insightful suggestions to improve the structure, flow and clarity of this paper.

Contributors AP involved in writing protocol, searches, screening, extraction, drafting of results and writing of manuscripts. VL and TB involved in content expert input (methodology) and editing manuscripts. GR oversaw the project, assisted with screening, content expert input, drafting of results and editing of manuscripts.

Funding This work was supported by the Professor Leon Piterman AM PhD scholarship, Southern Academic Primary Care Research Group, Monash university.

Competing interests None declared.

Patient consent Not required.

Provenance and peer review Not commissioned; externally peer reviewed.

Data sharing statement Further details on studies included in this scoping review can be retrieved by contacting the corresponding author at annette.peart@monash. edu.

Open Access This is an Open Access article distributed in accordance with the Creative Commons Attribution Non Commercial (CC BY-NC 4.0) license, which permits others to distribute, remix, adapt, build upon this work non-commercially, and license their derivative works on different terms, provided the original work is properly cited and the use is non-commercial. See: http://creativecommons.org/ licenses/by-nc/4.0/

(c) Article author(s) (or their employer(s) unless otherwise stated in the text of the article) 2018. All rights reserved. No commercial use is permitted unless otherwise expressly granted.

\section{REFERENCES}

1. Schamess A, Foraker R, Kretovics M, et al. Reduced emergency room and hospital utilization in persons with multiple chronic conditions and disability receiving home-based primary care. Disabil Health J 2017;10:326-33.

2. Loignon C, Hudon C, Goulet É, et al. Perceived barriers to healthcare for persons living in poverty in Quebec, Canada: the EQUIhealThY project. Int J Equity Health 2015;14:4.

3. Ford JA, Wong G, Jones AP, et al. Access to primary care for socioeconomically disadvantaged older people in rural areas: a realist review. BMJ Open 2016;6:e010652.

4. Davy C, Harfield S, McArthur A, et al. Access to primary health care services for Indigenous peoples: A framework synthesis. Int J Equity Health 2016;15:163.

5. Levesque JF, Harris MF, Russell G. Patient-centred access to health care: conceptualising access at the interface of health systems and populations. Int J Equity Health 2013;12:18-19.

6. McColl MA, Aiken A, Schaub M. Do people with disabilities have difficulty finding a family physician? Int $J$ Environ Res Public Health 2015;12:4638-51.

7. Shi L, Lebrun-Harris LA, Daly CA, et al. Reducing disparities in access to primary care and patient satisfaction with care: the role of health centers. J Health Care Poor Underserved 2013;24:56-66.

8. Starfield B. Primary care: balancing health needs, services, and technology. New York: Oxford University Press, 1998.

9. Starfield B. Primary care: an increasingly important contributor to effectiveness, equity, and efficiency of health services. SESPAS report 2012. Gac Sanit 2012;26:20-6.

10. Janamian T, Jackson CL, Glasson N, et al. A systematic review of the challenges to implementation of the patient-centred medical home: lessons for Australia. Med J Aust 2014;201:69-73.

11. Epstein RM, Fiscella K, Lesser CS, et al. Why the nation needs a policy push on patient-centered health care. Health Aff 2010;29:1489-95.

12. Kelly E, Ivers N, Zawi R, et al. Patient navigators for people with chronic disease: protocol for a systematic review and meta-analysis. Syst Rev 2015;4:28.

13. Natale-Pereira A, Enard KR, Nevarez L, et al. The role of patient navigators in eliminating health disparities. Cancer 2011;117:3541-50.

14. Jean-Pierre P, Hendren S, Fiscella K, et al. Understanding the processes of patient navigation to reduce disparities in cancer care: 
perspectives of trained navigators from the field. $J$ Cancer Educ 2011;26:111-20.

15. Dennis S, Hasan I, Jackson Pulver L, et al. Experiences and views of a brokerage model for primary care for Aboriginal people. Aust Health Rev 2015;39:26-32.

16. Freeman HP. The origin, evolution, and principles of patient navigation. Cancer Epidemiol Biomarkers Prev 2012;21:1614-7.

17. Manderson B, McMurray J, Piraino E, et al. Navigation roles support chronically ill older adults through healthcare transitions: a systematic review of the literature. Health Soc Care Community 2012;20:113-27.

18. Glick SB, Clarke AR, Blanchard A, et al. Cervical cancer screening, diagnosis and treatment interventions for racial and ethnic minorities: a systematic review. J Gen Intern Med 2012;27:1016-32.

19. Tho PC, Ang $E$. The effectiveness of patient navigation programs for adult cancer patients undergoing treatment: a systematic review. JBI Database System Rev Implement Rep 2016;14:295-321.

20. Chh T, Wilson S, McConigley R. Experiences of cancer patients in a patient navigation program: a qualitative systematic review. JBI Database System Rev Implement Rep 2015;13:136-68.

21. Robinson-White $\mathrm{S}$, Conroy $\mathrm{B}$, Slavish $\mathrm{KH}$, et al. Patient navigation in breast cancer: a systematic review. Cancer Nurs 2010;33:127-40.

22. Naylor K, Ward J, Polite BN. Interventions to improve care related to colorectal cancer among racial and ethnic minorities: a systematic review. J Gen Intern Med 2012;27:1033-46.

23. Genoff MC, Zaballa A, Gany F, et al. Navigating language barriers: a systematic review of patient navigators' impact on cancer screening for limited english proficient patients. J Gen Intern Med 2016:31:426-34.

24. Eschiti V, Burhansstipanov L, Watanabe-Galloway S. Native cancer navigation: the state of the science. Clin J Oncol Nurs 2012;16:73-82.

25. Ranaghan C, Boyle K, Meehan M, et al. Effectiveness of a patient navigator on patient satisfaction in adult patients in an ambulatory care setting: a systematic review. JBI Database System Rev Implement Rep 2016;14:172-218.

26. Meredith SM. Disparities in breast cancer and the role of patient navigator programs. Clin J Oncol Nurs 2013;17:54-9.

27. Krok-Schoen JL, Oliveri JM, Paskett ED. Cancer Care Delivery and Women's Health: The Role of Patient Navigation. Front Oncol 2016;6:2.

28. Valaitis RK, Carter N, Lam A, et al. Implementation and maintenance of patient navigation programs linking primary care with communitybased health and social services: a scoping literature review. BMC Health Serv Res 2017:17:116.

29. Institute of Medicine. Crossing the quality chasm: a new health system for the 21st century. Washington, DC: National Academies Press, 2001

30. Epstein RM, Street RL. The values and value of patient-centered care. Ann Fam Med 2011:9:100-3.

31. Sidani S, Fox M. Patient-centered care: clarification of its specific elements to facilitate interprofessional care. $J$ Interprof Care 2014;28:134-41.

32. Constand MK, MacDermid JC, Dal Bello-Haas V, et al. Scoping review of patient-centered care approaches in healthcare. BMC Health Serv Res 2014;14:1-9.

33. Cadzow RB, Craig M, Rowe J, et al. Transforming community members into diabetes cultural health brokers: the Neighborhood Health Talker project. Diabetes Educ 2013;39:100-8.

34. Torres S, Spitzer DL, Labonté R, et al. Community health workers in Canada: innovative approaches to health promotion outreach and community development among immigrant and refugee populations. J Ambul Care Manage 2013;36:305-18.

35. Arksey H, O'Malley L. Scoping studies: towards a methodological framework. Int J Soc Res Methodol 2005;8:19-32.

36. Levac D, Colquhoun H, O'Brien KK. Scoping studies: advancing the methodology. Implement Sci 2010;5:1-9.

37. Wang ML, Gallivan L, Lemon SC, et al. Navigating to health: Evaluation of a community health center patient navigation program. Prev Med Rep 2015;2:664-8.
38. Parker VA, Lemak $\mathrm{CH}$. Navigating patient navigation: crossing health services research and clinical boundaries. Adv Health Care Manag 2011;11:149-83.

39. Griswold KS, Homish GG, Pastore PA, et al. A randomized trial: are care navigators effective in connecting patients to primary care after psychiatric crisis? Community Ment Health J 2010;46:398-402.

40. Griswold KS, Servoss TJ, Leonard KE, et al. Connections to primary medical care after psychiatric crisis. J Am Board Fam Pract 2005;18:166-72.

41. Griswold KS, Zayas LE, Pastore PA, et al. Primary care after psychiatric crisis: a qualitative analysis. Ann Fam Med 2008;6:38-43.

42. Chan TC, Killeen JP, Castillo EM, et al. Impact of an internetbased emergency department appointment system to access primary care at safety net community clinics. Ann Emerg Med 2009;54:279-84.

43. Elliott K, W Klein J, Basu A, et al. Transitional care clinics for followup and primary care linkage for patients discharged from the ED. Am J Emerg Med 2016;34:1230-5.

44. Wexler R, Hefner JL, Sieck C, et al. Connecting emergency department patients to primary care. J Am Board Fam Med 2015;28:722-32.

45. Kangovi S, Mitra N, Grande D, et al. Patient-centered community health worker intervention to improve posthospital outcomes: a randomized clinical trial. JAMA Intern Med 2014;174:535-43.

46. Freeman HP. The history, principles, and future of patient navigation: commentary. Semin Oncol Nurs 2013;29:72-5.

47. Freeman HP, Rodriguez RL. History and principles of patient navigation. Cancer 2011;117:3537-40.

48. Gunn CM, Clark JA, Battaglia TA, et al. An assessment of patient navigator activities in breast cancer patient navigation programs using a nine-principle framework. Health Serv Res 2014;49:1555-77.

49. Bishop SE, Edwards JM, Nadkarni M. Charlottesville Health Access: a locality-based model of health care navigation for the homeless. $J$ Health Care Poor Underserved 2009;20:958-63.

50. Marr AL, Pillow T, Brown S. Southside medical homes network: linking emergency department patients to community care. Prehosp Disaster Med 2008;23:282-4.

51. Gany F, Bari S, Gill P, et al. Step on it! Impact of a workplace New York City taxi driver health intervention to increase necessary health care access. Am J Public Health 2015;105:786-92.

52. Overholser LS, Hassell K, Nuss R, et al. Using patient navigators to help adults with sickle cell disease obtain a primary care home. $J$ Clin Outcomes Manage 2014;21:304-7.

53. Kim TY, Mortensen K, Eldridge B. Linking uninsured patients treated in the emergency department to primary care shows some promise in Maryland. Health Aff 2015;34:796-804.

54. Treadwell $\mathrm{H}$, Holden $\mathrm{K}$, Hubbard R, et al. Addressing obesity and diabetes among African American men: examination of a communitybased model of prevention. J Natl Med Assoc 2010;102:794-802.

55. ED navigators connect patients to better venues of care. ED Manag 2011;23:53-5.

56. Aantjes C, Quinlan T, Bunders J. Integration of community home based care programmes within national primary health care revitalisation strategies in Ethiopia, Malawi, South-Africa and Zambia: a comparative assessment. Global Health 2014;10:851.

57. Doran KM, Colucci AC, Hessler RA, et al. An intervention connecting low-acuity emergency department patients with primary care: effect on future primary care linkage. Ann Emerg Med 2013;61:312-21.

58. Horwitz SM, Busch SH, Balestracci KM, et al. Intensive intervention improves primary care follow-up for uninsured emergency department patients. Acad Emerg Med 2005;12:647-52.

59. ED navigators help patients find a PCP. Hosp Case Manag 2014;22:9-10.

60. Parker VA, Clark JA, Leyson J, et al. Patient navigation: development of a protocol for describing what navigators do. Health Serv Res 2010;514+.

61. Kahn LS, Aiello J, Berdine DE, et al. The use of telephonic case management to link a special-needs population with a primary care physician. J Am Board Fam Med 2009;22:585-7. 